\title{
Hygiene Standards for Tattooists
}

\section{Andy Schmidt}

2nd Chairman DOT e.V., Willich-Neersen, Germany

\begin{abstract}
The following excerpt is taken from the hygiene guidelines written by Deutsche Organisierte Tätowierer (DOT e.V. Germany; German Association of Professional Tattoo Artists) and United European Tattoo Artists e.V. (UETA). It has been published with the intention of creating a standard that is understandable and accomplishable in practice, focusing on a minimum standard level that guarantees the highest possible safety for tattooists and customers at the same time. The DOT and UETA consistently strive to participate in the research of tattoo hygiene and tattoo colours because important insider information can be provided by professional tattoo artists with many years of work experience.
\end{abstract}

() 2015 DOT/UETA

The following hygiene guidelines have been developed by the Deutsche Organisierte Tätowierer (DOT; German Association of Professional Tattoo Artists) and United European Tattoo Artists e.V. (UETA) especially for tattoo studios. The main intention was to create a standard that is easily understandable and easily applicable in practice, focusing on a minimum standard level that guarantees $100 \%$ safety for tattooists and customers at the same time. Presented here is the version from 2008. Based on this version, we are currently developing a Comité Européen de Normalisation (European Committee for Standardisation) norm for Europe assisted by the Deutsches Institut für Normung (German Institute for Standardisation); therefore, there may be possible alterations in the cooperation with further European partners.

Since its creation in 1995, the DOT has continuously tried to improve the image of the art of tattooing. To a large extent, this has included the creation of hygienic standards and also cooperation with the relevant authorities. Many local health authorities in Germany adhere to our standards. Further, the DOT has participated in the Arbeitsgemeinschaft industrieller Forschungsvereinigungen (Consortium of Industrial Research, Collective Research Networking). Within this cooperation, hygiene has been evaluated in tattoo studios in Germany, Belgium and Austria, and the first tests on tattoo colours have been carried out.

The DOT is a founding member of the European Society of Tattoo and Pigment Research. The DOT consistently strives to participate in the research of tattoo colours specifically. However, tattooing should not be dealt with only under medicine or cosmetics, it needs to be considered under a special status. Unfortunate- 
ly, there is little data to review. Research is important but requires funding that neither the DOT nor the manufacturers of tattoo colours can supply.

\section{Excerpt from the 'Hygiene Standards for Tattooists'}

As of May 2008. All rights reserved by the DOT and UETA.

The unauthorised copying, duplication, and reproduction of this document or its parts are prohibited.

\section{Health Risks and Infections during Tattooing}

The process of tattooing poses a theoretical risk of infection with viruses transmittable through the blood, although this risk has not been verified with scientific data. Even a mathematical implication of the data at hand is inconclusive. According to the literature, the prevalence of tattooing in the German population is $10 \%$; hence, approximately 8 million persons are tattooed in Germany. Many have more than 1 tattoo and have large tattoos that require several sessions to complete. If we multiply these 8 million tattoos by a factor of 2.5 , we are talking about 20 million tattoo sessions in Germany. If only $1 \%$ of these sessions resulted in infectious complications, a total of 200,000 infections would have occurred. Assuming those 20 million tattoo sessions took place during the last 10 years and assuming a $1 \%$ infection rate, in Germany alone, 20,000 infections would have been contracted annually through tattooing. Even at a $0.1 \%$ infection rate, there would still be 2,000 cases per year.

\section{Vocational Training}

Individuals who apply tattoos must have basic knowledge in the following areas:

- general and specific microbiology, germinal sources and ways of transmission, causative organisms of skin and wound infections, and vi- ruses transmitted by the blood, especially hepatitis $B$ virus (HBV), hepatitis $\mathrm{C}$ virus (HCV) and human immunodeficiency virus (HIV)

- general hygiene (germinal sources and ways of transmission and possibilities and methods of preventing transmission

- specific hygiene (cleaning, disinfection and sterilisation, the treatment of instruments relevant to the transmission of pathogenic germs, hand disinfection, skin disinfection, surface disinfection, protection against re-contamination, the handling of sterile materials, water requirements, the handling of disposables, and waste disposal)

- personal protection (disposable medical gloves, protective gear, the washing and disinfection of hands and clothing, and the prevention of environmental contamination)

- procedures that support the fast healing of wounds sustained through tattooing

\section{Work Area}

The work area has to be easily cleanable, well ventilated and adequately lit. Flooring and work surfaces must be smooth and water repellent and/or easy to wipe and disinfect (e.g. tiles, polyvinyl chloride, and linoleum). A washstand should be close to the work area but placed in such a location that the contamination of the work area through splashing or aerosols may not take place.

The following equipment is needed:

- a hand disinfectant dispenser

- a liquid soap dispenser

- paper towels

- a waste disposal container

A smooth and easy-to-clean work space for setting down instruments is required. The work space should be covered with disposables (e.g. foil and paper towels). All furniture and fixtures (tattoo chair/arm rest) must have smooth and easyto-clean and easily disinfectable surfaces. If necessary, liquid-repelling or impermeable disposable covers have to be used to cover chairs and gurneys. 
If disposable materials are not used exclusively, equipment must be available to prepare and treat instruments and objects (cleaning, disinfection, and sterilisation) on site.

\section{Sterilisation and Autoclaving}

Sterilisation is safer because it leaves materials cleaner and lowers contamination levels. Through prior cleaning and disinfection, the initial germ count should be reduced to a low level. Manufacturer's specifications concerning concentration and disinfection and sterilisation times must be followed to inactivate or kill microorganisms on or inside of the processed materials to eliminate their infectivities if the materials possess a common grade of contamination. Dry heat is sufficient for sterilising grips and needle holders.

\section{Used Needle Disposal}

Standard commercial needle disposal containers for used needles must be available. The container has to stay securely shut, and once full, it must be disposed of.

\section{Tattoo Machines}

Tattoo machines and clip cords have to be freshly bagged for each customer. Commercial plastic bags, such as freezer bags and plastic wrap, are adequate. After tattooing, bags must be removed, and instruments must be cleaned and disinfected with an appropriate agent. Instruments must be disconnected from their power sources.

\section{Materials and Instruments Needed}

- for operator safety, disposable medical gloves (non-sterile) must be available in adequate quantities

- an appropriate alcohol-based hand disinfectant has to be in stock if possible as well as an attached dispenser or a pump bottle

- for skin disinfection prior to tattooing, an alcohol-based disinfectant must be used. (Note: this is considered the use of an approved drug, therefore, make sure to strictly follow the manufacturer's instructions)

- disposable cleaning cloths/tissues for cleaning the work space (work table, tattoo chair, and arm rest). Disposable paper towels can be used.

- an alcohol-based surface disinfectant for the disinfection of small instruments

- a surface disinfectant for flooring and appropriate towels

- disposable tattoo needles

- if necessary, disposable needle jigs and grips

If disposable needles, needle jigs and grips are used exclusively, additional resources for the treatment of the instruments are not required.

- disposable bowls for inks (separate ones for each customer)

- an ultrasonic cleaner to clean the needle jigs and grips

- a disinfectant for instruments that is effective against $\mathrm{HBV}, \mathrm{HCV}$ and $\mathrm{HIV}$

- a disinfectant pan

- running water to rinse instruments (of drinking-water quality)

- if necessary, a steriliser

- appropriate packaging material for sterilised needle jigs

- a closed storage system (lockable drawers and cabinets) to prevent the contamination of sterilised materials

- quality control to ensure for the efficiency of the sterilisation procedures

\section{Colours}

Ink bottles must be stored closed and protected from dust to prevent microorganisms from contaminating the contents. Colours have to comply with current tattoo guidelines.

The following information must be included on the ink bottle label:

- 'for tattoo purpose' or 'tattoo ink' or 'tattoo colour'

- lot number

- manufacturer information 
- expiration date

- storage life after opening bottle

- list of ingredients

\section{Disinfection Procedures}

\section{Hands}

Hand disinfection is one of the most important measures to prevent infections. The washing of hands should be reduced to a minimum. In cases of an increased risk of infection or contamination, especially while handling customer's excretions or objects contaminated with blood or sputum, the wearing of disposable medical gloves to protect the hands is mandatory. Contaminated hands may be washed with water and soap only after proper disinfection. Hand disinfection is mandatory in the following situations:

- after contact with blood, secretions or excretions

- before physical contact with customers or before touching the skin area to be tattooed

- after contact with contaminated surfaces or objects

- after taking off disposable gloves

Skin

Objective: To attain an adequate reduction of germs on the respective skin area prior to the procedure. The shaving of large areas of skin to be tattooed has to be done directly before beginning the tattoo session, and a suitable disinfectant has to be sprayed on with an exposure time of at least 15 seconds. Only original containers with the manufacturer's labels may be used. Do not decant.

\section{Preparation of Hygiene-Relevant Equipment}

Using the same colours for different customers and using reprocessed needles are the main risks in tattooing. Therefore, using new, disposable ink cups and new, disposable needles for each cus- tomer are the most important and effective hygienic measures.

Because needle jigs may be contaminated with blood, they may also be contaminated with viruses transmitted through the blood, and because under certain circumstances, jigs may be in direct contact with the skin, another major risk of transferring relevant microorganisms is related to the use of reusable needle jigs. Additional increased safety can be achieved by using disposable needle jigs.

If disposable needle jigs are used exclusively, preparation and sterilisation procedures are redundant (see preliminary note on sterilisation).

Contamination of the tattoo machine during or because of its use cannot be ruled out. Therefore, a theoretical possibility of cross-contamination exists. The disinfection of tattoo machines is limited, and sterilisation is not possible at all.

\section{Preparation of Needle Jigs and Grips}

Risk Assessment

- no direct contact with blood, contamination with blood and ink are possible and likely

- effective cleaning and quality control of cleaning efficiency is possible

- only dismountable needle jigs and grips should be used, with components that do not have significant air pockets to impact sterilisation

- treatments must follow proper and controlled procedures

\section{Measures}

- cleaning, disinfection and sterilisation after each usage

- locked storage in appropriate packaging (lockable cases, drawers, and cabinets)

- removal only immediately prior to use

- if necessary, assembly prior to use

- the placement of materials and instruments on disposable paper towels or foil

\section{Preparation for Treatment}

After Usage: Temporary storage on disposable paper towels, disassembly into its parts only while 
wearing non-sterile disposable gloves, and the placement of components into an appropriate container (to disinfect or dispose of them).

\section{Cleaning}

Thorough cleaning is a precondition for the effectiveness of later disinfection and sterilisation processes. Adherent blood and ink residues may significantly impact disinfection and sterilisation.

Non-disinfected components, especially sharp and pointed objects, bear the risk of injury and the infection of personnel. Protective disposable gloves must be worn while handling contaminated objects.

Because of hard-to-remove pigment particles, particularly when they are mixed with blood, it is recommended to subject such contaminated objects to an ultrasonic cleaning procedure. Ultrasonic cleaning procedures should only be applied to the components. The ultrasonic bath must be filled with disinfectant solution, which allows for the effective inactivation of HBV/HCV/HIV.

Furthermore, the instrument disinfectant agent must be suitable for ultrasonic cleaning. The change of the disinfectant agent must take place according to the manufacturer's instructions daily or earlier depending on the level of contamination (and for longer periods only with a certificate provided by the manufacturer).

Inspection for Residues

A thorough visual inspection with sufficient lighting and, if necessary, the use of a magnifying glass ensures that objects are free of residue (clean). Particular attention must be paid to hollow spaces, edges and corners. If needed, hollow spaces should be checked against the light. If necessary, targeted cleaning and drying procedures should be repeated, followed by inspection.
These were abstracts of the paper 'Hygiene standards for tattooists'.

The entire paper can be seen on: http://www. ueta.org/service/guidelines/.

Hygiene standards established by:

- Heribert Nentwig, (former) Chairman UETA e.V., (former) Hygiene Supervisor DOT.e.V. c/o Tattoo-Center Koblenz, Triererstrasse 38, DE-56072 Koblenz (Germany)

- Andy Schmidt, 2nd Chairman DOT e.V., Hauptstrasse 60, DE-47877 Willich-Neersen (Germany)

Hygiene standards verified and revised by:

- Prof. Dr. med. Burkard Wille, Arzt für Hygiene und Umweltmedizin und Arzt für Mikrobiologie und Infektionsepidemiologie, iki Institut für Krankenhaushygiene Gbr., Siemensstrasse 18, DE-35394 Giessen (Germany), Tel.: +49641979050

Physician for Hygiene and Environmental Medicine and Physician for Micro-Biology and Infectious Epidemiology:

- Prof. Dr. med. Burkard Wille, Siemensstrasse 18, DE-35394 Giessen (Germany)

Tel.: +49641979050

General contact DOT e.V. (Deutsche Organisierte Tätowierer $=$ German Association of professional tattoo artists)

- www.dot-ev.de, 2nd Chairman Andy Schmidt, Hauptstr. 60, DE-47877 Willich-Neersen (Germany)

Tel.: +4921565240

General contact UETA e.V. (United European Tattoo Artists)

- www.ueta.org, Chairman Maik Frey, Sirnauer Strasse 23, DE-73728 Esslingen (Germany)

Tel. +497115357277

Andy Schmidt

2nd Chairman DOT e.V.

Hauptstrasse 60

DE-47877 Willich-Neersen (Germany)

E-Mail andysbodyelectric@yahoo.de 